\title{
Control Mechanisms for the Growth of Isolated Vertically Aligned Carbon Nanofibers
}

\author{
Vladimir I. Merkulov, ${ }^{*}, \dagger$ D. K. Hensley ${ }^{\dagger}$ A. V. Melechko, ${ }^{\dagger}, \star$ M. A. Guillorn,,$\uparrow \S$ \\ D. H. Lowndes," and M. L. Simpson ${ }^{\dagger, \#, \$}$
}

Molecular Scale Engineering and Nanoscale Technologies (MENT) and Thin Film and Nanostructured Materials Physics (TFNMP) Groups, Oak Ridge National Laboratory, Oak Ridge, Tennessee 37831, and

Department of Materials Science and Engineering (DMSE) and Center of Environmental Biotechnology (CEB), University of Tennessee, Knoxville, Tennessee 37996

Received: February 14, 2002; In Final Form: July 1, 2002

\begin{abstract}
Isolated vertically aligned carbon nanofibers (VACNFs) have been grown using dc plasma-enhanced chemical vapor deposition, and the effects of the growth conditions on VACNF morphology and composition have been determined in substantial detail. The dependence of the growth rate, tip and base diameters, and chemical composition of isolated VACNFs on the growth parameters is described, including the effects of plasma power and gas mixture. Phenomenological models explaining the observed growth behavior are presented. The results indicate the importance of plasma control for the deterministic growth of isolated VACNFs, which are promising elements for the fabrication of practical nanoscale devices.
\end{abstract}

\section{Introduction}

"Forests" of vertically aligned carbon nanofibers (VACNFs) can be synthesized using plasma-enhanced chemical vapor deposition (PECVD), which was first demonstrated by Ren et al. ${ }^{1}$ However, deterministic growth ${ }^{2}$ of isolated VACNFs is required to make them attractive for various applications. Deterministic growth of VACNFs implies the ability to control their location, height, tip diameter, shape, orientation, and chemical composition during a large-scale synthesis process ${ }^{2-6}$ and thereby to enable fabrication of practical devices. ${ }^{7-9}$ VACNFs similar to those synthesized by our group often are referred to in the literature as carbon nanotubes. However, we prefer the term "nanofibers" because, despite the superficial external morphological similarity between VACNFs and multiwalled carbon nanotubes (MWCNTs), the crystalline structures are quite different. Whereas MWCNTs consist of concentric graphene cylinders, VACNFs are composed of graphitic "funnels" and cones, also referred to as a "herringbone" and "bamboo-like" structures. Schematic representations of the structures of MWCNTs and CNFs are shown in Figure 1. Thus, the difference between VACNFs and MWCNTs is not simply the higher degree of crystallinity of MWCNTs, but essentially different long-range crystalline structures. As a result, the physical and chemical properties of VACNFs are expected to be quite different from those of MWCNTs, so that clear differentiation is highly desirable. Previously, Nolan et al. also emphasized the importance of such differentiation. ${ }^{10}$ Even VACNFs with graphitic planes nearly parallel to the fiber axis (small angle $\alpha$; Figure 1a), which often gives them a gross appearance similar to that of MWCNTs, should have properties that are substantially different from those of true MWCNTs composed of graphene cylinders $(\alpha=0)$.

* To whom all correspondence should be addressed. Electronic mail merkulovvi@ornl.gov.

†ENT, Oak Ridge National Laboratory.

$\div$ CEB, University of Tennessee.

$\S$ DMSE, University of Tennessee.

" TFNMP, Oak Ridge National Laboratory. (a)

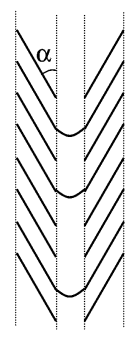

VACNF (b)

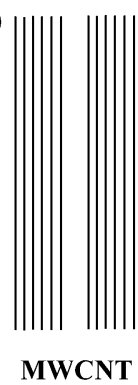

Figure 1. Schematic representation of the crystalline structures of (a) a carbon nanofiber and (b) a multiwalled carbon nanotube.

The synthesis of VACNFs involves many deposition parameters, such as plasma power, gas mixture, temperature, pressure, etc., and each of them can have a profound effect on the growth and properties of the resultant VACNFs. On the other hand, the ability to control properties is essential for device fabrication and operation. To date, there have been a number of reports by different groups that investigated PECVD growth of VACNFs. ${ }^{1-6,11-19}$ (Hereafter, the term VACNFs includes conically shaped VACNFs, which are also referred to as vertically aligned carbon nanocones in some of our previous work. ${ }^{5}$ ) Despite recent progress, the VACNF synthesis process is sufficiently complex that more research is needed before all aspects of it are well understood. In the present work, we study VACNF growth in more detail, discuss new aspects of this growth, and show how systematic changes in different growth parameters lead to controllable modifications of VACNF properties, which is especially important for the realization of practical devices.

\section{Experimental Section}

VACNFs were grown using direct-current (dc) plasmaenhanced chemical vapor deposition (PECVD). The vacuum chamber was evacuated before growth to a base pressure of $\sim 1 \times 10^{-5}$ Torr. Nickel (Ni) catalyst dots $100 \mathrm{~nm}$ in diameter and $40 \mathrm{~nm}$ thick were used to produce isolated VACNFs and 


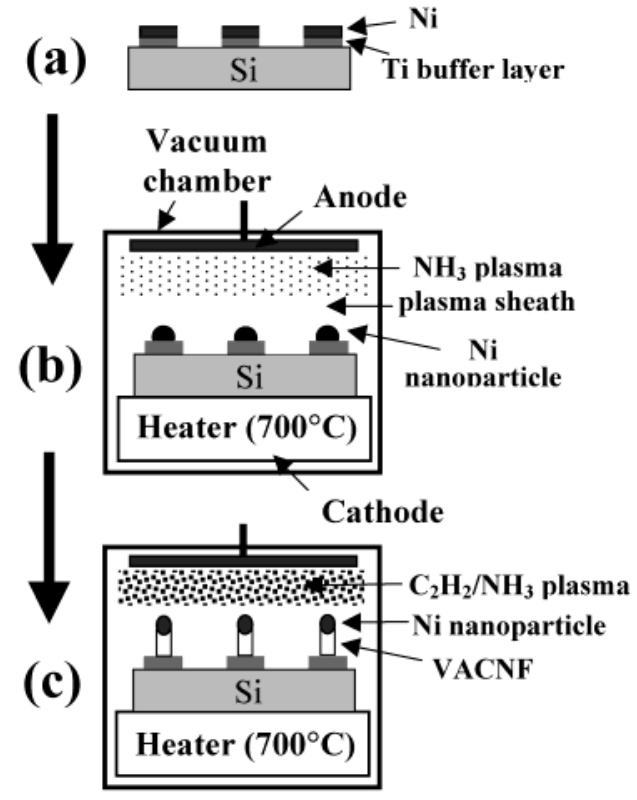

Figure 2. Schematic representation of the PECVD process for growing vertically aligned carbon nanofibers.

were prepared on Si substrates using conventional electron-beam lithography. A 10-nm-thick Ti layer was deposited between the $\mathrm{Ni}$ catalyst and the $\mathrm{Si}$ substrate to prevent catalyst silicide formation at the moderately high growth temperature of $\sim 700$ ${ }^{\circ} \mathrm{C}$. The sample was mounted directly on a heater plate, which served as a cathode (Figure 2a). After the chamber had reached the base pressure, ammonia $\left(\mathrm{NH}_{3}\right)$ was introduced into the chamber, and the sample was pretreated with the $\mathrm{NH}_{3}$ plasma and temperature. As a result of this treatment, catalyst nanoparticles were formed from the deposited catalyst dots (Figure 2b). ${ }^{2}$ For Ni catalyst dots with the diameter and thickness used here, only a single nanoparticle is formed from each catalyst dot. These nanoparticles act as the necessary seeds for the catalytic growth of isolated VACNFs. ${ }^{20}$ After the pre-etching step, with the $\mathrm{NH}_{3}$ plasma still on, acetylene $\left(\mathrm{C}_{2} \mathrm{H}_{2}\right)$ was introduced into the chamber, and the VACNF growth began (Figure 2c). The VACNF tip diameter is approximately equal to that of the nanoparticle. The initial diameter of nanoparticles, $d$, is roughly determined from mass conservation of the catalyst, that is, $1 / 6 \pi d^{3}=1 / 4 \pi D^{2} t$ or $d=\left(3 / 2 t D^{2}\right)^{1 / 3}$, where $D$ is the diameter of the catalyst dot and $t$ is its thickness. However, $d$ decreases as the growth proceeds. ${ }^{5}$ The $\mathrm{NH}_{3}$ gas flow was maintained at a constant level of $80 \mathrm{sccm}$, and the $\mathrm{C}_{2} \mathrm{H}_{2}$ gas flow was varied to produce different gas mixtures. The total gas pressure during growth was $\sim 3$ Torr.

Post-growth imaging of the VACNFs was carried out using a Hitachi S4700 high-resolution scanning electron microscope equipped with an X-ray energy dispersive spectroscopy (EDS) detector (Oxford Instruments 7200). Imaging was performed at $10 \mathrm{kV}$ and a $45^{\circ}$ sample tilt. EDS measurements were performed by focusing the electron beam on the middle part of individual VACNFs to minimize contributions from the catalyst particle and the buffer layer. The sample tilt during the measurements was $20^{\circ}$, and the electron beam energy was 5 $\mathrm{keV}$ to provide for better surface sensitivity. Typically, peaks due to carbon $(\mathrm{C})$, nitrogen $(\mathrm{N})$, oxygen $(\mathrm{O})$, and silicon $(\mathrm{Si})$ were observed. The oxygen signal was attributed to adsorbed water. It was present mainly in nitrogenated VACNFs, which we believe is due to the hydrophilic nature of the surface of such nanofibers, and its concentration typically did not exceed

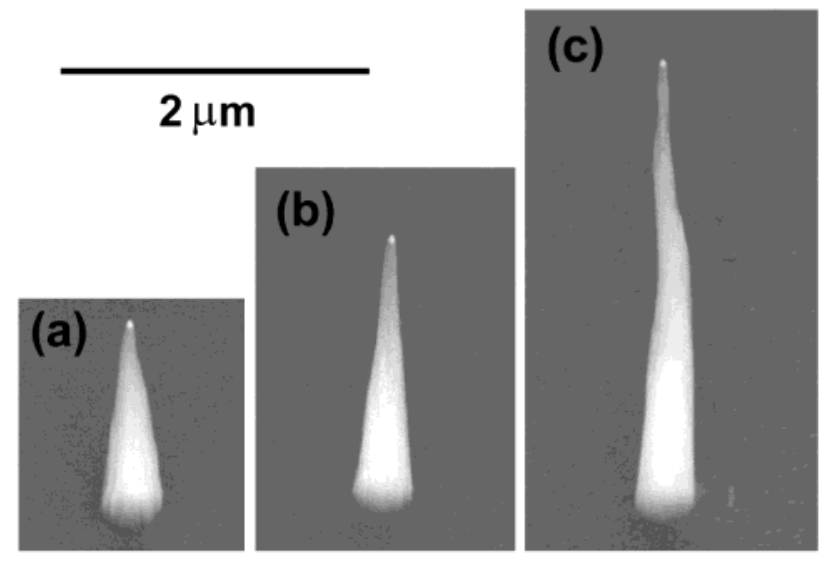

Figure 3. SEM images of isolated VACNFs prepared with a dc plasma power of $\sim 123 \mathrm{~W}(200 \mathrm{~mA})$ and $\mathrm{C}_{2} \mathrm{H}_{2} / \mathrm{NH}_{3}$ gas ratios of (a) 0.813 , (b) 0.788 , and (c) 0.688 . The growth time for all VACNFs was $30 \mathrm{~min}$.

a few atomic percent. The $\mathrm{Si}$ peak was attributed to the $\mathrm{Si}$ substrate. The $\mathrm{N}$ fractional concentration within the VACNFs was calculated as the ratio between the measured $\mathrm{N}$ content and the sum of $\mathrm{C}, \mathrm{N}$, and $\mathrm{O}$ contents, i.e., $\mathrm{N}$ fractional content $=\mathrm{N} /(\mathrm{C}+\mathrm{N}+\mathrm{O})$, all in atomic percent. We note that the analysis software (INCA 3.01) assumes a flat, homogeneous, and thick sample to perform the elemental analysis. This is quite different from the sample configuration that we used, i.e., a tilted nanometer-scale nanofiber standing vertically on a flat $\mathrm{Si}$ substrate. However, we found the calculated $\mathrm{N}$ content to be essentially independent of the tilt angle when varied between $0^{\circ}$ and $30^{\circ}$ and very similar (within a few percent) for all VACNFs from a given sample. Also, the results for different electron beam energies $(2-10 \mathrm{keV})$ varied within only $\sim 5 \%$. Finally, our samples were measured using a second independent EDS system, and essentially identical values for the elemental composition were obtained. Therefore, we believe that our EDS results are fairly accurate and can serve as a reasonably good estimate of the $\mathrm{N}$ content within VACNFs.

\section{Results and Discussion}

A. Gas Flow Ratio. In previous work, we showed that the $\mathrm{C}_{2} \mathrm{H}_{2} / \mathrm{NH}_{3}$ gas flow ratio has a profound effect on the shape ${ }^{4}$ and chemical composition ${ }^{6}$ of isolated VACNFs. In particular, lower $\mathrm{C}_{2} \mathrm{H}_{2} / \mathrm{NH}_{3}$ ratios lead to smaller cone angles of VACNFs and also to the incorporation of significant amounts of nitrogen into the nanofibers. Clearly, this is related to a corresponding change in the composition of the carbonaceous and etchant species present in the glow discharge, which results in VACNFs with different properties. In the present work, we find that, for isolated VACNFs in the regime of near-optimal growth conditions, the reduction of the $\mathrm{C}_{2} \mathrm{H}_{2} / \mathrm{NH}_{3}$ gas ratio results in an increased growth rate. Figure 3 shows isolated VACNFs prepared at different gas ratios but otherwise identical growth conditions. One can see that not only the cone angle but also the length of the VACNFs is dependent on the $\mathrm{C}_{2} \mathrm{H}_{2} / \mathrm{NH}_{3}$ gas ratio: the smaller the ratio, the taller the nanofibers. The systematic variation of the VACNF growth rate as a function of $\mathrm{C}_{2} \mathrm{H}_{2}$ content is shown in Figure 4. Chhowalla et al. ${ }^{15}$ observed a similar effect in the growth of dense forests of VACNFs, in which case the increase in $\mathrm{C}_{2} \mathrm{H}_{2}$ content resulted in reduced growth rates above some critical value for the gas ratio.

At first sight, this dependence seems somewhat surprising because a higher $\mathrm{C}_{2} \mathrm{H}_{2}$ content is associated with a higher carbon supply and consequently (one might assume) with a higher 


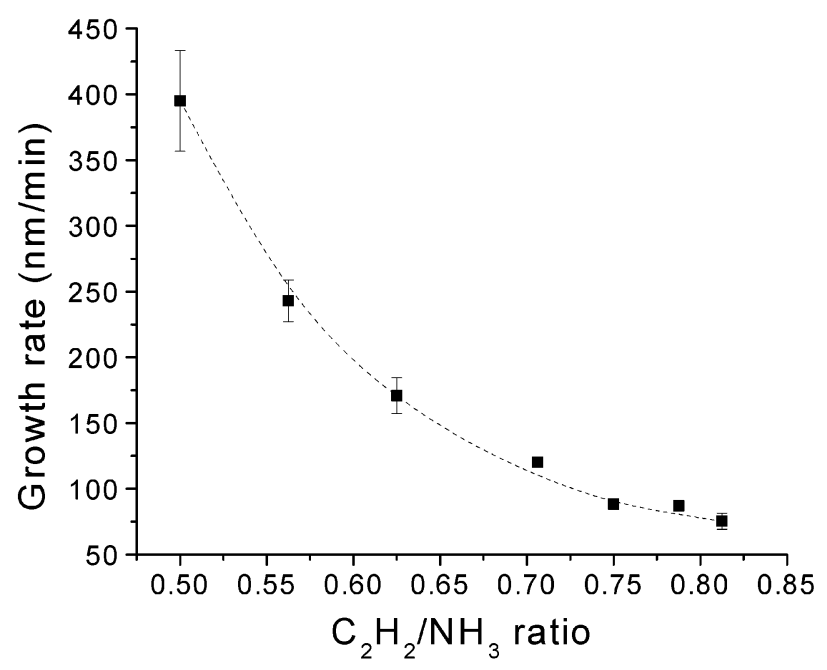

Figure 4. VACNF growth rate (defined as final length/growth time) as a function of $\mathrm{C}_{2} \mathrm{H}_{2} / \mathrm{NH}_{3}$ ratio. The plasma power was held constant at $\sim 123 \mathrm{~W}(200 \mathrm{~mA})$. Hereafter, the error bars indicate the standard error obtained from averaging 5-10 VACNFs for each growth run.

growth rate. However, more careful consideration suggests an explanation that is consistent with our present understanding of nanofiber growth. The "classical" model for the mechanism of nanofiber growth by conventional thermal CVD was first proposed by Baker et al. and later was discussed and refined by several other research groups (see refs 20 and 21 and references therein). According to this model, the growth occurs in three main steps: (i) decomposition of the carbon-containing molecules at the catalyst nanoparticle surface, (ii) diffusion of free carbon atoms through the nanoparticle, and (iii) precipitation of diffused carbon at the catalyst/nanofiber interface. Because the rate at which the carbonaceous molecules decompose at the catalyst surface is one of the growth-rate-determining factors, a possible explanation for the change in the VACNF growth rate is that changing the gas ratio shifts the chemical distribution of carbon-containing species toward species that decompose at a different rate at the nanoparticle surface. In particular, in a certain gas ratio range, despite the fact that increasing the $\mathrm{C}_{2} \mathrm{H}_{2}$ content produces more carbonaceous species, the chemical distribution of the excited species might change such that more of these species catalytically decompose at a lower rate, with the net result being slower growth. We note that the above mechanism implies that the VACNF growth rate is supplylimited, not diffusion-limited.

A change in the chemical composition of the species that are present in the plasma and serve as feedstock for the growth of VACNFs is confirmed by EDS analysis of chemical composition of the VACNFs. Examples of the EDS spectra and the nitrogen content within VACNFs as a function of the $\mathrm{C}_{2} \mathrm{H}_{2} /$ $\mathrm{NH}_{3}$ gas ratio are shown in Figure 5a and $b$, respectively. Clearly, the $\mathrm{N}$ content increases as the $\mathrm{C}_{2} \mathrm{H}_{2} / \mathrm{NH}_{3}$ gas ratio decreases, consistent with a change in the chemical composition of the species that are present in the glow discharge and form the VACNFs. A very interesting observation is that, according to the EDS analysis, at low values of the gas ratio, VACNFs can contain extraordinarily high amounts of nitrogen, up to $\sim 70 \%$. In fact, these nanofibers should more properly be referred to as nitrogen nanofibers containing carbon. Although, at first sight, such a high $\mathrm{N}$ content might seem somewhat suspicious, it is comparable to the results obtained for some carbon nitride films, in which case the $\mathrm{N}$ content was found to be as high as $\sim 60 \%$. $^{22}$ In fact, nitrogen-rich carbon nitride films
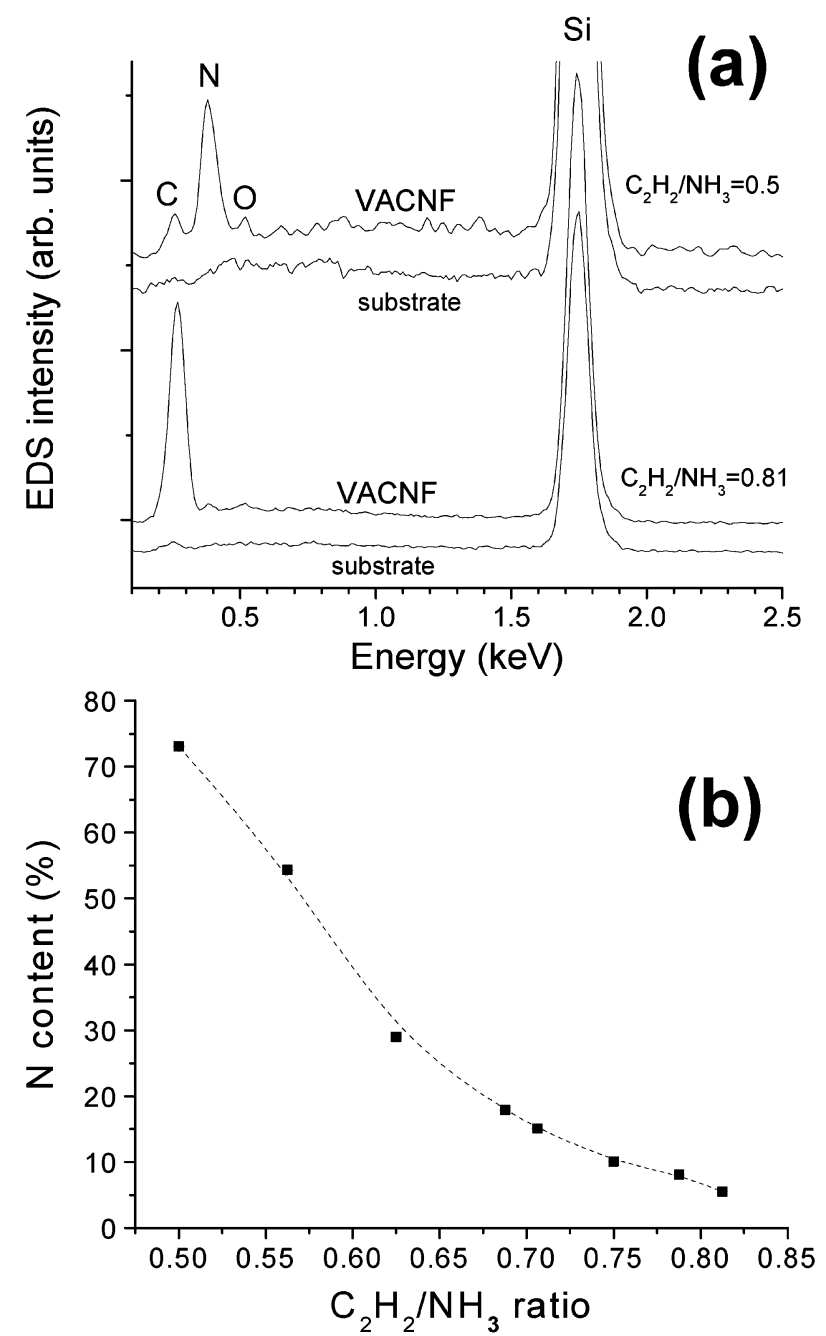

Figure 5. (a) EDS spectra of VACNFs and corresponding substrates for the two growth runs with $\mathrm{C}_{2} \mathrm{H}_{2} / \mathrm{NH}_{3}$ ratios of 0.500 (upper spectra) and 0.813 (lower spectra). (b) Nitrogen content within VACNFs, derived from EDS measurements, as a function of the $\mathrm{C}_{2} \mathrm{H}_{2} / \mathrm{NH}_{3}$ gas ratio. The plasma power for all growth runs was kept constant at $\sim 123 \mathrm{~W}(200$ $\mathrm{mA})$.

can be synthesized by a PECVD technique that is very similar to that used to grow VACNFs. In any case, even if substantial uncertainty in the absolute values of the $\mathrm{N}$ content cannot be completely excluded, the relative $\mathrm{N}$ content and the general trend shown in Figure 5 should remain valid. More work is under way to confirm the results obtained by EDS and further elucidate the structure and properties of these interesting nanofibers.

There is, however, another possible explanation for the decreased growth rate at increased $\mathrm{C}_{2} \mathrm{H}_{2} / \mathrm{NH}_{3}$ gas ratios. It was reported previously $2,6,15,17$ that the catalyst becomes covered with a film of carbon if the carbonaceous gas content is too high during PECVD growth, thus preventing the diffusion of free carbon atoms into the nanoparticle and consequently stopping VACNF growth. In thermal CVD, the analogous phenomenon occurs and is especially pronounced if the growth temperature is too high, which leads to high decomposition rates and subsequent formation of a carbon shell (many monolayers of carbon) around the catalyst particle. However, a regime should exist in which the $\mathrm{C}$ film just starts to form but is not yet continuous, as shown schematically in Figure 6. At lower $\mathrm{C}_{2} \mathrm{H}_{2}$ contents (Figure 6a), only a few $\mathrm{C}$ patches are present on the catalyst surface. Consequently, the clean catalyst area available for decomposition of carbonaceous gas molecules is quite large, 


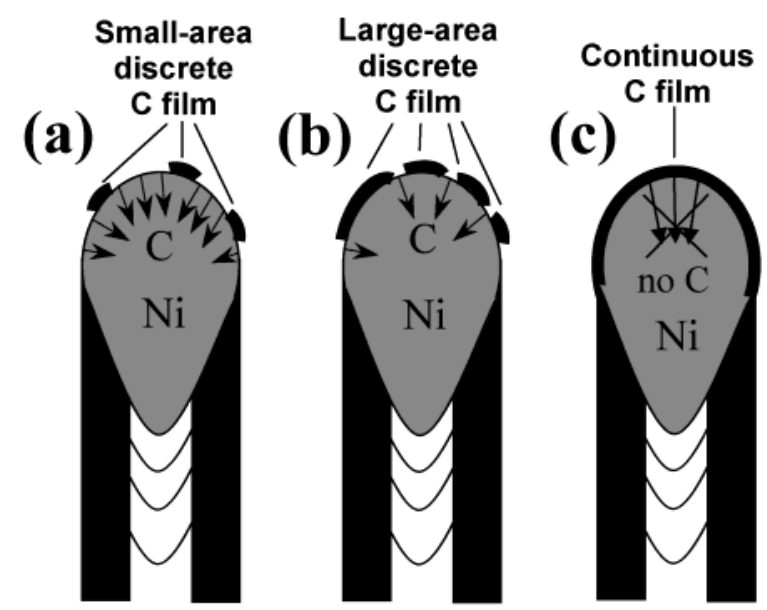

Figure 6. Schematic representation of the formation of an initially discrete ( $\mathrm{a}$ and $\mathrm{b}$ ) and later continuous (c) carbon shell around the catalyst nanoparticle as the $\mathrm{C}_{2} \mathrm{H}_{2}$ content is gradually increased from (a) lower to (b) higher and to (c) yet higher values.

and the growth rate is high. If the $\mathrm{C}_{2} \mathrm{H}_{2}$ content is increased (Figure 6b), the $\mathrm{C}$ islands increase in size and number, which reduces the area of clean catalyst surface and leads to fewer decomposed $\mathrm{C}$ atoms and consequently a lower growth rate. Of course, the actual situation is dynamic, i.e., the islands emerge and disappear, and it is the time-average covered and clean catalyst surface areas that we must consider. If the $\mathrm{C}_{2} \mathrm{H}_{2}$ content is increased even further (Figure 6c), the nanoparticle is covered with a continuous $\mathrm{C}$ film, and the growth is stopped. To summarize this model, changing the $\mathrm{C}_{2} \mathrm{H}_{2}$ content changes the area of the catalyst surface covered by the $\mathrm{C}$ film, controlling the number of decomposed carbon species and thereby the growth rate. This mechanism also suggests supply-limited growth. It might be that the two mechanisms just discussed coexist, with the former being more important at lower $\mathrm{C}_{2} \mathrm{H}_{2}$ contents and the latter at higher $\mathrm{C}_{2} \mathrm{H}_{2}$ contents.

The notion of supply-limited growth also is supported by recent results of Chhowalla et al., ${ }^{15}$ who showed that the activation energy for VACNF growth is significantly lower than that for carbon diffusion in nickel. ${ }^{15}$ We note that equality of the two activation energies is found for conventional thermal CVD growth, ${ }^{23}$ which has been the basis for assuming that thermal CVD growth is diffusion-limited. The lower activation energy for the PECVD of VACNFs strongly suggests that the PECVD growth is not diffusion-limited. In our view, the most likely scenario is supply-limited growth, with the supply increasing at higher temperatures as a result of higher catalytic decomposition and yielding a correspondingly higher growth rate. Of course, if the temperature is too high, a thin carbon shell starts to form at the catalyst surface as a result of the high pyrolysis rate. Thus, above a critical growth temperature, the growth rate will decrease, as observed by Chhowalla et al.

For thermal CVD, the two activation energies for carbon diffusion in $\mathrm{Ni}$ and for VACNF growth are equal. ${ }^{23}$ This means that the supply rate is equal to or higher than the diffusion rate, but at the same time, the thin carbon shell is not being formed. To satisfy these two conditions, we propose that, in purely thermal CVD, above a certain temperature and concentration of the carbonaceous gas, a monolayer of free carbon atoms is always present at the catalyst surface (or perhaps a submonolayer if some regions of the catalyst surface are inactive). As soon as the surface carbon atom diffuses into the interior of the nanoparticle, another surface carbon atom quickly forms in its place as a result of thermal catalytic decomposition. The decomposition rate should be higher than the diffusion rate to maintain this monolayer of carbon at the nanoparticle surface. In thermal CVD at moderate temperatures, the incoming carbonaceous atoms attach and decompose only at the bare catalyst surface, not at the carbon monolayer covering the surface, thus always yielding only a single monolayer of carbon. In contrast, in PECVD, the reactive carbonaceous species from the plasma can attach both to the catalyst and to the carbon already on its surface. Consequently, a carbon shell can easily form if the supply of the carbonaceous species is sufficiently high. Of course, the carbon shell can form even in thermal CVD at high temperatures, for which the pyrolysis rate becomes high and substantial decomposition of the carbonaceous molecules starts to occur at the carbon monolayer as well.

The idea of supply-limited growth of VACNFs during PECVD is further supported by the fact that the growth rate of isolated VACNFs using smaller nanoparticle sizes was not found to be higher than that of VACNFs grown using larger nanoparticles. For example, when we grew VACNFs from 50-nm catalyst dots, the growth rate was actually lower $(\sim 25 \%)$ than it was for 100-nm dots. Clearly, if the diffusion rate were the limiting factor, the growth rate would be higher for the smaller particles. We note that our results differ from those obtained by Bower et al. ${ }^{13}$ and Chhowalla et al., ${ }^{15}$ who indeed found a higher growth rate for smaller nanoparticle sizes. However, in the work by Bower et al., the catalyst nanoparticles were located at the nanofiber base. In this case, the growth mechanism is similar to that of thermal CVD because, as a result of the electric field line distribution and physical shielding by the neighboring VACNFs, ${ }^{6}$ the charged reactive species from the plasma strike essentially only the tip and the upper part of the VACNFs, not the base where the catalyst nanoparticle is located. The VACNF base is exposed to mainly nonreactive species, similarly to thermal CVD. As discussed earlier, thermal CVD is a diffusionlimited process, and therefore, the growth rate increases with decreasing particle size, precisely as observed by Bower et al. The increased growth rate with the decreasing nanoparticle size in the work by Chhowalla et al. might occur due to fact the that, for different forests of VACNFs, different local plasma conditions and consequently different species in the discharge can form, which can lead to different growth rates. This mechanism will be discussed in more detail in section $\mathrm{C}$ below.

B. Plasma Power. The profound effect that the plasma power can have on the growth of VACNFs was first demonstrated by Huang et al., ${ }^{11}$ who found that the VACNF growth rate increases substantially with increasing plasma power. This is an interesting and important observation for both the fundamental and practical aspects of VACNF growth. However, understanding a direct relationship between the increased growth rate and the plasma power in the above experiments is not straightforward. Other parameters such as the substrate temperature and the catalyst nanoparticle size also were changed along with the plasma power. In addition, the effective chemical composition of the species present in the plasma also might change with the plasma power. Consequently, direct assignment of the observed increase in the growth rate to the increased plasma power does not reveal much about the underlying growth mechanisms.

To obtain a better understanding of the effects of plasma power on the growth of VACNFs, we investigated this aspect of the growth process in substantially more detail. Also, we conducted our experiments using well-separated VACNFs, the growth of which can be substantially different from that of largearea dense VACNF forests. ${ }^{6}$ We find that, if the gas ratio is kept constant while the plasma power is changed, both the 


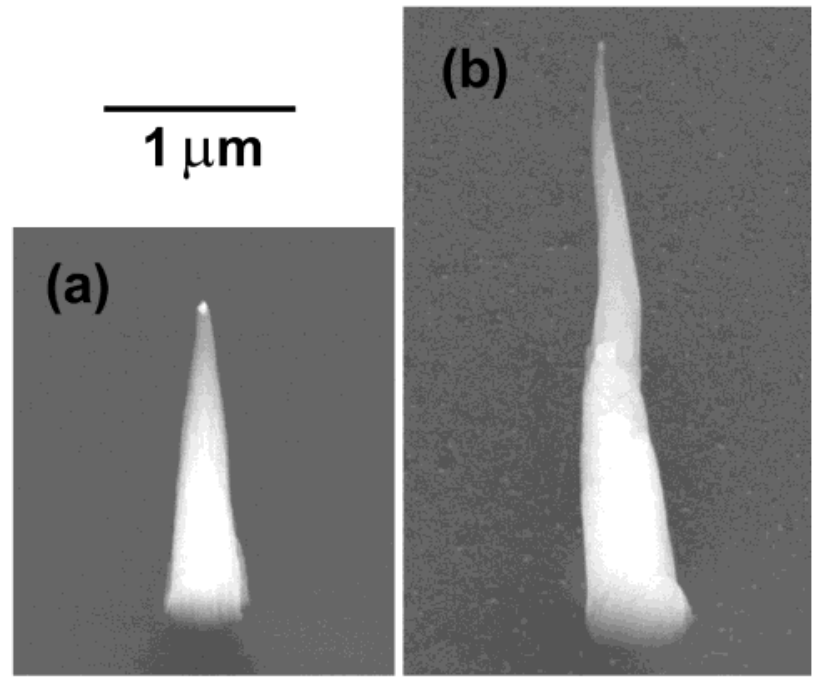

Figure 7. SEM images of VACNFs prepared at plasma powers of (a) $\sim 66 \mathrm{~W} / 120 \mathrm{~mA}$ and $(\mathrm{b}) \sim 208 \mathrm{~W} / 300 \mathrm{~mA}$. The $\mathrm{C}_{2} \mathrm{H}_{2} / \mathrm{NH}_{3}$ ratio was kept constant at $\sim 0.706$, and the growth time was 30 min for both a and $\mathrm{b}$.

morphology and the chemical composition of the resultant VACNFs change as well, as shown in Figure 7. It must be mentioned that the plasma current and voltage can have quite different effects on the growth of VACNFs but in a dc glow discharge these are coupled and cannot be changed independently. For instance, increasing the plasma power increases both the current and the voltage. Thus, in the following discussion we will present values for both the plasma power and current.

As the plasma power is increased, VACNFs clearly experience a higher growth rate, as can be seen from Figure 7, and their $\mathrm{N}$ content also is significantly increased, e.g., from just a few percent for plasma power of $\sim 66 \mathrm{~W}(120 \mathrm{~mA})$ (Figure 7a) to $\sim 30 \%$ for $\sim 208 \mathrm{~W}$ (300 mA) (Figure $7 \mathrm{~b}$ ). These changes are quite similar to those observed when the gas ratio is changed because, as was discussed above, a reduced gas ratio also leads to higher growth rates and higher $\mathrm{N}$ contents. Therefore, we suggest that the main effect of changing the plasma power is actually to change the distribution of the chemical composition of the species in the glow discharge and that it is these compositional changes that lead to the increased nitrogen incorporation and growth rate of the resultant VACNFs, in agreement with our previous observations.

To test this hypothesis, we adjusted the gas ratio to compensate for the possible change in the chemical composition of the glow discharge that is caused by changing the plasma power. The objective was to synthesize cones of similar geometries (cone angles) using different plasma power levels and observe any changes in the growth rate and chemical composition. We found that, indeed, it is possible to produce VACNFs with very similar cone angles, such as those shown in Figure 8, using quite different plasma power levels, if the $\mathrm{C}_{2} \mathrm{H}_{2}$ content is increased along with increasing the power. Moreover, the height of these VACNCs was essentially constant regardless of the plasma power used (Figure $8 b-f$ ). The VACNC growth rate started to decrease only at very low plasma power, below $44 \mathrm{~W}$. A "dandruff-like" structure also started to form on the sidewalls of VACNCs at $\sim 44 \mathrm{~W}$ plasma power and became well-developed at $\sim 23 \mathrm{~W}$, as shown in Figure 8a. This probably occurred because physical-chemical etching of the VACNC sidewalls is quite small at low plasma power, which leads to the formation of the dandruff-like superstructures that otherwise are etched away at higher plasma powers.
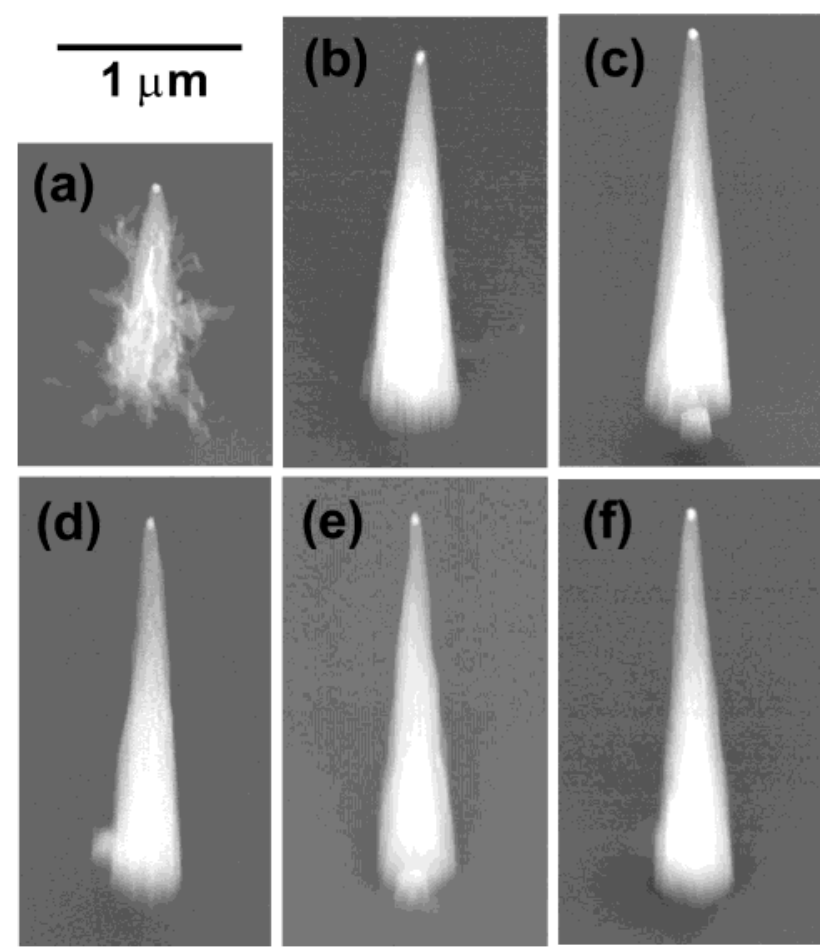

Figure 8. SEM images of VACNFs prepared at plasma powers of (a) $23 \mathrm{~W} / 50 \mathrm{~mA}$, (b) $44 \mathrm{~W} / 85 \mathrm{~mA}$, (c) $66 \mathrm{~W} / 120 \mathrm{~mA}$, (d) $123 \mathrm{~W} / 200 \mathrm{~mA}$, (e) $208 \mathrm{~W} / 300 \mathrm{~mA}$, and (f) $288 \mathrm{~W} / 400 \mathrm{~mA}$ and $\mathrm{C}_{2} \mathrm{H}_{2} / \mathrm{NH}_{3}$ ratios of (a) 0.625 , (b) 0.675 , (c) 0.706 , (d) 0.788 , (e) 0.888 , and (f) 1.000 . The growth time was $30 \mathrm{~min}$ for all VACNFs.

The $\mathrm{C}_{2} \mathrm{H}_{2} / \mathrm{NH}_{3}$ gas ratio required to produce VACNFs of similar geometries is shown as a function of plasma power and plasma current in Figure 9. Interestingly, both the current and power dependencies have an approximately linear form, indicating that, to produce similar VACNFs, the increase in plasma power should be matched by a corresponding increase in the $\mathrm{C}_{2} \mathrm{H}_{2} / \mathrm{NH}_{3}$ gas ratio. The plots in Figure 9 are important because they allow one to easily produce morphologically identical VACNFs at different plasma power levels simply by adjusting the $\mathrm{C}_{2} \mathrm{H}_{2}$ content in the gas mixture (at our fixed $\mathrm{NH}_{3}$ flow of $80 \mathrm{sccm})$. Furthermore, EDS measurements revealed that morphologically similar VACNFs produced at different plasma power levels, with scaling of the $\mathrm{C}_{2} \mathrm{H}_{2}$ flow according to Figure 9 , have very similar chemical compositions and consist of nearly pure carbon with only a few percent of nitrogen. These observations again strongly suggest that the main effect of changing the plasma power is to change the composition of species present in the glow discharge, which in turn changes the growth of the VACNFs.

C. Tip Diameter. The diameter of the catalyst nanoparticles at the tips of isolated VACNFs decreases with growth time, as was demonstrated in our previous work. ${ }^{5}$ This phenomenon is interesting from both scientific and technological points of view. It shows that the growth of isolated VACNFs cannot continue indefinitely because, at some point, the nanoparticles will disappear. When this happens, the VACNF growth stops, and destruction of the VACNFs by the plasma from the tip downward ensues. The practical aspect of this phenomenon is that reduction of the nanoparticle size is an effective process for sharpening the VACNFs, which is quite important for applications such as field emission and scanning probe microscopy. The continuous decrease of the nanoparticle size during VACNF growth was previously attributed to chemical-physical 

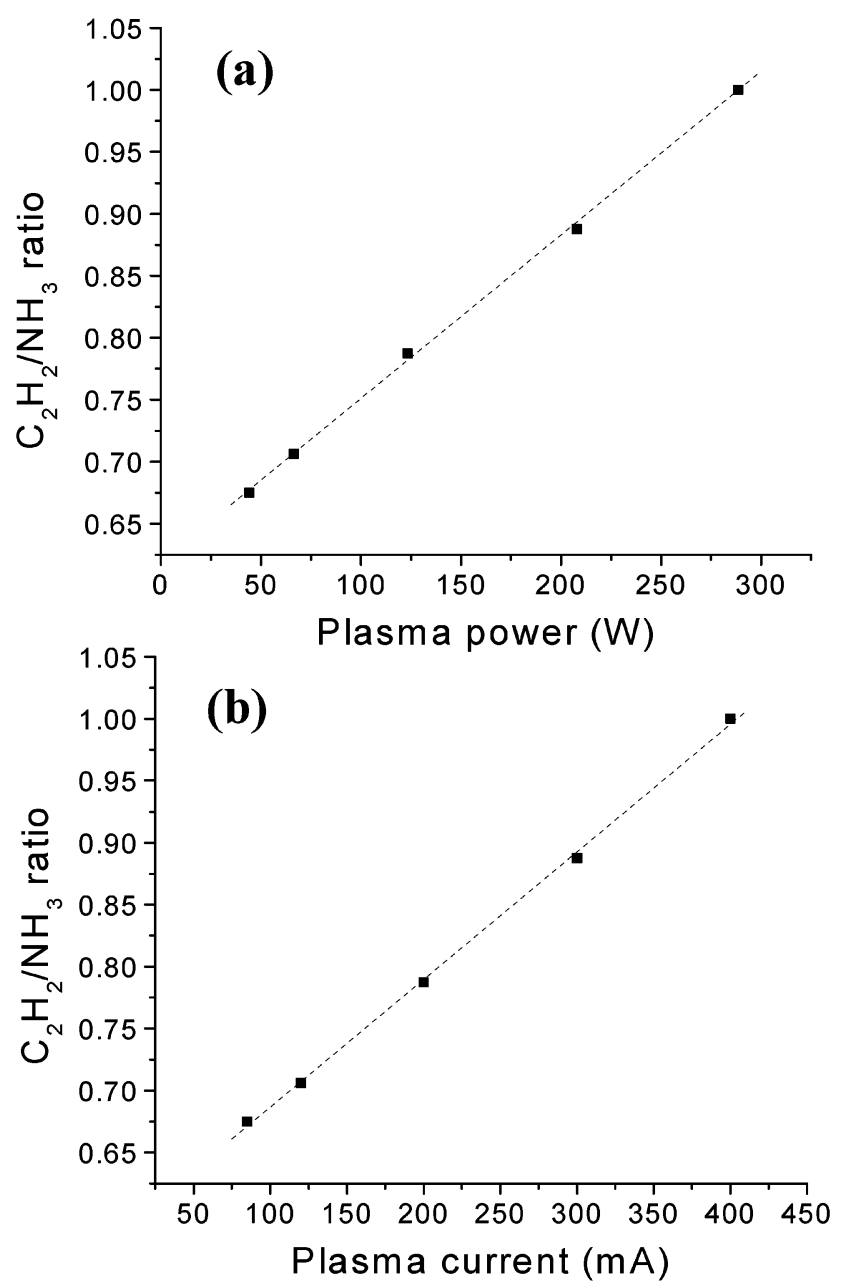

Figure 9. $\mathrm{C}_{2} \mathrm{H}_{2} / \mathrm{NH}_{3}$ ratio required to produce VACNFs similar to those shown in Figure $8 \mathrm{~b}-\mathrm{f}$ as a function of (a) plasma power and (b) plasma current.

sputtering due to the plasma and/or to gradual dispersion of the catalyst material over the VACNC body. ${ }^{5}$

Here, we further investigated the control of VACNF tip diameter. First, we measured the diameter of the nanoparticle as a function of VACNF height because there is often a substantial height variation among isolated VACNFs, even when they are produced under nominally identical growth conditions. No correlation was observed between the nanoparticle size and the VACNF height under the same growth conditions. This seems to indicate that dispersing the catalyst material over the body of VACNFs is not the principal factor in reducing the nanoparticle size because the catalyst dispersal would be expected to be proportional to the VACNF height. Large-area dense forests of VACNFs with initial nanoparticle sizes similar to that of isolated VACNFs also were found to grow to much greater heights than is possible for isolated VACNFs. Even though the local plasma conditions above the forests and isolated VACNFs can be different, as discussed below, this nevertheless indicates that catalyst dispersal is not the determining factor.

Second, we measured the diameter of the catalyst nanoparticles as a function of plasma power and current with the $\mathrm{C}_{2} \mathrm{H}_{2} /$ $\mathrm{NH}_{3}$ gas ratio adjusted according to Figure 9. The corresponding data are shown in Figure 10a and b. Even though the linear fit to the data indicates that a slight reduction in the catalyst nanoparticle size might occur as the plasma power/current is increased, no definite conclusion can be drawn because of the large error bars. From these observations, it is safe to conclude
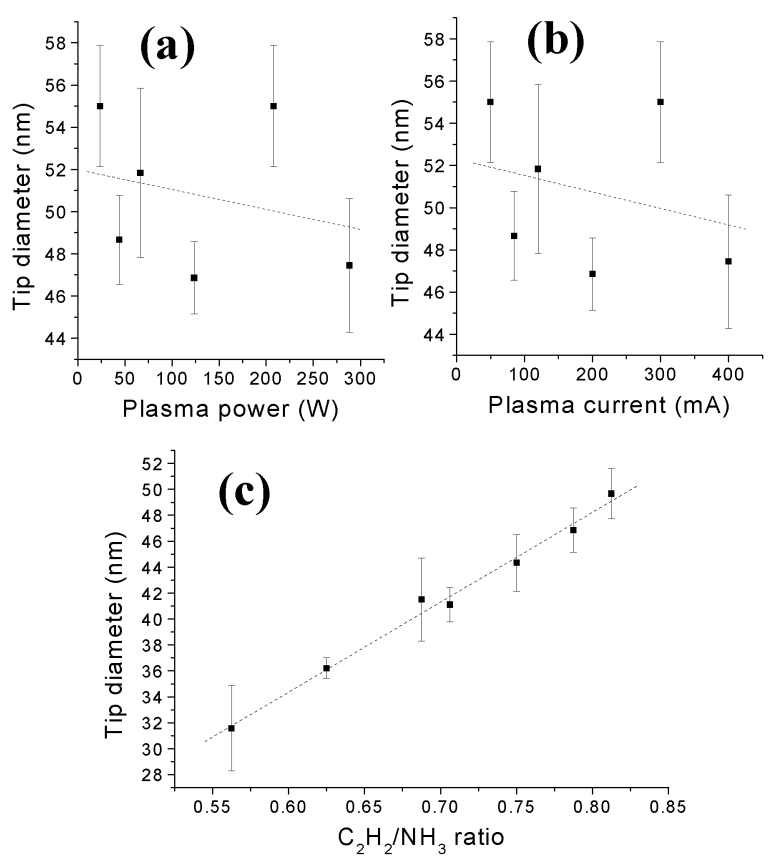

Figure 10. VACNF tip diameter as a function of (a) plasma power and (b) plasma current with $\mathrm{C}_{2} \mathrm{H}_{2}$ ratio changing as indicated in Figure 9 and as a function of (c) $\mathrm{C}_{2} \mathrm{H}_{2} / \mathrm{NH}_{3}$ ratio with plasma power being kept constant at $\sim 123 \mathrm{~W}(200 \mathrm{~mA})$. The growth time was $30 \mathrm{~min}$.

that physical sputtering is not likely to be a significant component in reducing the nanoparticle size. If it were, then a substantially higher sputtering yield would have been expected at higher plasma power because of the increased energy and the number of bombarding ions, but this is not observed.

However, we did observe a substantial reduction of the nanoparticle size/tip diameter with decreasing $\mathrm{C}_{2} \mathrm{H}_{2} / \mathrm{NH}_{3}$ gas ratio at constant plasma power and growth time, as shown in Figure 10c. This strongly suggests that the dominant mechanism for nanoparticle reduction is chemical etching of the catalyst metal. As the gas ratio is decreased, more reactive species are created, and these species etch the catalyst particle more effectively. As was noted earlier, dense, large-area VACNF forests with initially similar catalyst nanoparticle sizes will grow much taller than individual VACNFs regardless of the gas ratio used. We believe that this occurs because, in addition to the plasma power and gas ratio, the material below the plasma also affects which species are present in the plasma immediately above the material's surface. This can happen as a result of different secondary electron yields as well as different electric field distributions for different materials. In the case of isolated VACNFs, the determining material is the substrate on which the VACNFs are grown, i.e., $\mathrm{Si}$ in the present work. For dense VACNF forests, however, the determining material is the VACNFs themselves. Therefore, the species in the plasma immediately above a dense VACNF forest, and consequently the etching rate, will be different as compared to those present above isolated VACNFs. As was mentioned earlier, a much smaller etching rate is observed for the forests.

D. Base Diameter. VACNF base diameter is an important parameter in the synthesis of VACNFs by PECVD. As we demonstrated previously, ${ }^{4,6}$ the base diameter of isolated VACNFs in most cases is larger than the tip diameter, in which case the VACNFs are referred to as carbon nanocones (VACNCs). On the practical side, the VACNF base determines mechanical and thermal stability of VACNFs. From the synthesis point of view, the base diameter is a measure of the lateral VACNF 


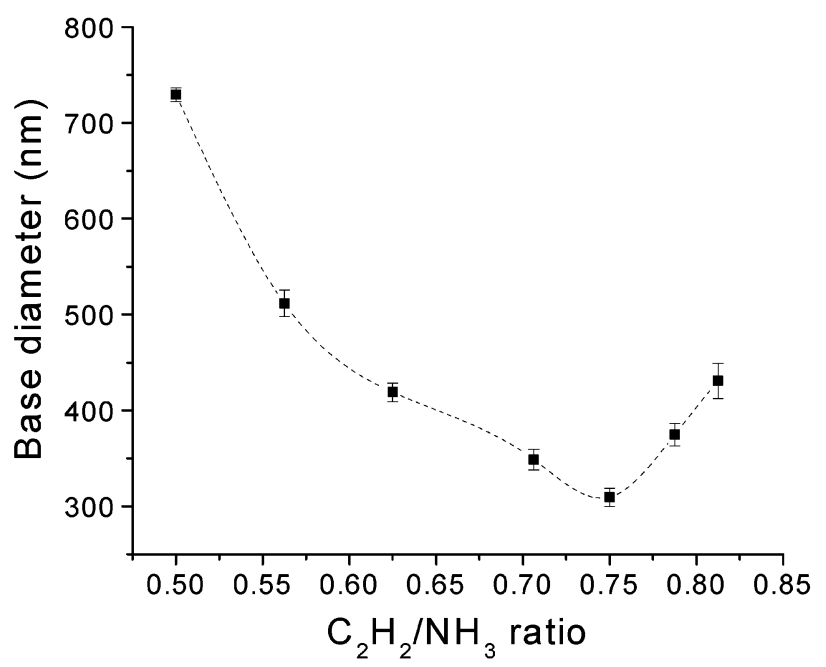

Figure 11. Base diameter as a function of the $\mathrm{C}_{2} \mathrm{H}_{2} / \mathrm{NH}_{3}$ ratio. The plasma power was kept constant at $\sim 123 \mathrm{~W}(200 \mathrm{~mA})$, and the growth time was $30 \mathrm{~min}$.

growth that occurs during PECVD concurrent with its upward growth by the nanoparticle-mediated catalytic mechanism. The presence of quasi-two-dimensional growth of VACNFs in PECVD in directions perpendicular (catalytic) and parallel (due to the reactive species from the plasma) to the substrate 4 is in contrast to conventional thermal CVD, where, in the absence of high-temperature pyrolysis, only quasi-one-dimensional catalytic growth occurs.

We recently reported that the VACNC cone angle is a function of the gas ratio ${ }^{4,6}$ and can be decreased by decreasing the $\mathrm{C}_{2} \mathrm{H}_{2}$ content. Here, we studied the base diameter as a function of the gas ratio, with the results shown in Figure 11.As the $\mathrm{C}_{2} \mathrm{H}_{2}$ concentration is decreased (or the $\mathrm{NH}_{3}$ concentration is increased) the base diameter initially decreases $\left(\mathrm{C}_{2} \mathrm{H}_{2} / \mathrm{NH}_{3}\right.$ $\approx 0.75-0.82$ in Figure 11 ). This can easily be explained by the fact that fewer carbonaceous species and/or more etchant species being created, so that less carbon is deposited on the VACNC sidewalls as a result of heavier etching and/or higher consumption of the carbonaceous species by the etchant species in the gas phase. However, when the $\mathrm{C}_{2} \mathrm{H}_{2}$ concentration is reduced further $\left(\mathrm{C}_{2} \mathrm{H}_{2} / \mathrm{NH}_{3} \leq 0.75\right)$, the base diameter again starts to increase. This suggests that the growth process is too complex to be described accurately using only the two "parameters" carbonaceous species derived from $\mathrm{C}_{2} \mathrm{H}_{2}$ and etchant species derived from $\mathrm{NH}_{3}$. In reality, "mixed" species must be present that contain both carbon and nitrogen and do not behave as a etchants but are incorporated into the lateral growth. Consequently, further reduction of the $\mathrm{C}_{2} \mathrm{H}_{2}$ concentration leads to the increased formation of these mixed (nonetchant) species that can participate in the lateral growth of VACNFs. The EDS measurements of the chemical composition of VACNFs that were described earlier (see Figure 5) support this conclusion as well. The measurements show that the $\mathrm{N}$ content within the VACNFs increases with decreasing $\mathrm{C}_{2} \mathrm{H}_{2} / \mathrm{NH}_{3}$ ratio, which supports the presence of the mixed species. Clearly, more studies are needed to identify the etchant and feedstock species that are present in the plasma during VACNF growth.

\section{Conclusions}

In conclusion, we have shown that the PECVD growth of vertically aligned carbon nanofibers is a complex process governed by interconnected growth parameters. Despite this complexity, a number of growth trends were identified, and a high degree of control was established over the important nanofiber properties. We demonstrated how the nanofiber growth rate, tip and base diameters, and chemical composition all can be controlled by altering the growth conditions. This is quite important for practical applications for which controlled synthesis of nanofibers is essential for device fabrication. The importance of plasma conditions as a determining factor in the resultant nanofiber properties cannot be overemphasized. However, little is known at this time about the actual species that are created in the glow discharge and subsequently reach the substrate surface. More research involving emission and absorption spectroscopy and mass spectrometry measurements will be very helpful in elucidating this issue, which is key to a more complete understanding, and subsequently more complete control, of nanofiber properties.

Acknowledgment. We thank Dr. David Joy for useful discussions, Pam Fleming for help with sample preparation, and Alla Landa for continuous encouragement. This research was supported by the Laboratory Directed Research and Development Program of Oak Ridge National Laboratory (ORNL); by the Office of Basic Energy Sciences, Division of Materials Sciences, U.S. Department of Energy; and by the Defense Advanced Research Projects Agency under Contract $1868 \mathrm{HH} 26 \mathrm{X} 1$ with ORNL. The research was carried out at ORNL, managed by UT-Battelle, LLC, for the U.S. Department of Energy under Contract DE-AC05-00OR22725 and in part at the Cornell Nanofabrication Facility (a member of the National Nanofabrication Users Network), which is supported by the National Science Foundation under Grant ECS-9731293, its users, Cornell University, and Industrial Affiliates.

\section{References and Notes}

(1) Ren, Z. F.; Huang, Z. P.; Xu, J. W.; Wang, J. H.; Bush, P. S.; Siegal, M. P.; Provencio, P. N. Science 1998, 282, 1105-1107.

(2) Merkulov, V. I.; Lowndes, D. H.; Wei, Y. Y.; Eres, G.; Voelkl, E Appl. Phys. Lett. 2000, 76, 3555-3557.

(3) Ren, Z. F.; Huang, Z. P.; Wang, D. Z.; Wen, J. G.; Xu, J. W.; Wang, J. H.; Calvet, L. E.; Chen, J.; Klemic, J. F.; Reed, M. A. Appl. Phys. Lett. 1999, 75, 1086-1088.

(4) Merkulov, V. I.; Guillorn, M. A.; Lowndes, D. H.; Simpson, M. L.; Voelkl, E. Appl. Phys. Lett. 2001, 79, 1178-1180.

(5) Merkulov, V. I.; Melechko, A. V.; Guillorn, M. A.; Lowndes, D. H.; Simpson, M. L. Chem. Phys. Lett. 2001, 350, 381-385.

(6) Merkulov, V. I.; Melechko, A. V.; Guillorn, M. A.; Lowndes, D H.; Simpson, M. L. Appl. Phys. Lett. 2001, 80, 476-478.

(7) Guillorn, M. A.; Melechko, A. V.; Merkulov, V. I.; Ellis, E. D.; Britton, C. L.; Simpson, M. L.; Lowndes, D. H.; Baylor, L. R. Appl. Phys. Lett. 2001, 79, 3506-3508.

(8) Pirio, G.; Legagneux, P.; Pribat, D.; Teo, K. B. K.; Chhowalla, M.; Amaratunga, G. A. J.; Milne, W. I. Nanotechnology 2001, 13, 1-4

(9) Guillorn, M. A.; McKnight, T. E.; Melechko, A. V.; Merkulov, V. I.; Britt, P. F.; Austin, D. W.; Lowndes, D. H.; Simpson, M. L. J. Appl. Phys. 2002, 91, 3824-3828.

(10) Nolan, P. E.; Lynch, D. C.; Cutler, A. H. J. Phys. Chem. B 1998 , $102,4165-4175$

(11) Huang, Z. P.; Xu, J. W.; Ren, Z. F.; Wang, J. H.; Siegal, M. P.; Provencio, P. N. Appl. Phys. Lett. 1998, 73, 3845-3847.

(12) Bower, C.; Zhu, W.; Jin, S.; Zhou, O. Appl. Phys. Lett. 2000, 77, $830-832$.

(13) Bower, C.; Zhou, O.; Zhu, W.; Werder, D. J.; Jin, S. Appl. Phys Lett. 2000, 77, 2767-2769.

(14) Cui, H.; Zhou, O.; Stoner, B. R. J. Appl. Phys. 2000, 88, 60726074.

(15) Chhowalla, M.; Teo, K. B. K.; Ducati, C.; Rupesinghe, N. L.; Amaratunga, G. A. J.; Ferrari, A. C.; Roy, D.; Robertson, J.; Milne, W. I J. Appl. Phys. 2001, 90, 5308-5317.

(16) Delzeit, L.; McAninch, I.; Cruden, B. A.; Hash, D.; Chen, B.; Han, J.; Meyyappan, M. J. Appl. Phys. 2002, 91, 6027-6033.

(17) Teo, K. B. K.; Chhowalla, M.; Amaratunga, G. A. J.; Milne, W. I.; Hasko, D. G.; Pirio, G.; Legagneux, P.; Wyczisk, F.; Pribat, D. Appl. 
Phys. Lett. 2001, 79, 1534-1536.

(18) Merkulov, V. I.; Melechko, A. V.; Guillorn, M. A.; Lowndes, D. H.; Simpson, M. L. Appl. Phys. Lett. 2001, 79, 2970-2972.

(19) Tanemura, M.; Iwata, K.; Takahashi, K.; Fujimoto, Y.; Okuyama, F.; Sugie, H.; Filip, V. J. Appl. Phys. 2001, 90, 1529-1533.
(20) Baker, R. T. K. Carbon 1989, 27, 315-323.

(21) Rodriguez, N. M. J. Mater. Res. 1993, 8, 3233-3250.

(22) Wang, E. G. Prog. Mater. Sci. 1997, 41, 241-298.

(23) Baker, R. T. K; Chludzinski, J. J., Jr. J. Catal. 1980, 64, 464- 